\title{
Comportamento Térmico, Mecânico e Morfológico de Compósitos de Polietileno de Alta Densidade Reciclado com Fibra de Piaçava
}

\author{
Claudia M. C. Bonelli, A. Elzubair, João C. Miguez Suarez \\ Departamento de Engenharia Mecânica e de Materiais, IME
}

\author{
Eloisa B. Mano \\ Instituto de Macromoléculas Professora Eloisa Mano, UFRJ
}

\begin{abstract}
Resumo: O uso de fibras naturais como reforçadores para termoplásticos tem despertado interesse crescente, principalmente para termoplásticos reciclados, devido ao baixo custo, utilização de recursos naturais brasileiros disponíveis, além de contribuir para a despoluição ambiental. No presente trabalho foram avaliadas as propriedades térmicas, mecânicas e morfológicas de compósitos de polietileno de alta densidade reciclado (HDPE-r) e fibra de piaçava da Amazônia (Leopoldinia piassaba), tratada e não-tratada, em diferentes proporções (5, 10 e 15\% de fibra). O trabalho tem como objetivo a obtenção de compósitos a fim de atender as exigências do mercado como materiais alternativos, visando à fabricação de placas de baixo custo para estruturas sob pequeno esforço. As fibras foram submetidas a tratamento superficial com silano de duas formas: impregnação direta (fibra-ts) e com solução metanólica (fibra-tms). Foi verificado que os compósitos HDPE-r / fibra-ts apresentaram melhor desempenho mecânico que os obtidos com fibra-tms, em função da remoção pelo metanol da fração contendo lignina, componente estrutural da fibra. O aumento da adesão fibra-matriz de HDPE reciclado em compósitos com fibra tratada, conforme observado pelas microfratografias apresentadas, pode explicar este desempenho. A estabilidade térmica dos compósitos foi pouco afetada com a incorporação da fibra tratada.
\end{abstract}

Palavras-chave: Compósitos poliméricos, piaçava, polietileno de alta densidade reciclado.

Thermal, Mechanical and Morphological Behavior of Recycled High- Density Polyethylene Reinforced with Piassava Fiber

Abstract: The use of natural fibers as reinforcement for thermoplastics, especially for recycled thermoplastics, has generated much interest due to their low cost, possibility of environmental protection and use of locally available renewable resources. In this work we evaluate the thermal, mechanical and morphological properties of composites of recycled high-density polyethylene (HDPE-r) with treated and untreated piassaba fibers (Leopoldinia piassaba) in different proportions $(5,10$ and $15 \% \mathrm{w} / \mathrm{w}$ ). The objective of this work is to obtain composites of HDPE-r / piassaba fiber in order to fulfill the needs of the consumer market, aiming at preparing low-cost plates for light duty jobs. Fibers were submitted to superficial treatment with silane in two different ways: direct incorporation and treatment with methanolic solution of the coupling agent. The results show that composites of HDPE with silane-treated-only fiber had better mechanical performance than methanolic solutions of silane; the increase on fiber - polymer matrix interface adhesion, as revealed from morphological analysis, can explain this higher performance. Thermal stability of the composites is not greatly affected with the addition of treated fiber.

Keywords: Polymer composites, piassaba fibers, recycled high density polyethylene.

\section{Introdução}

A presença de embalagens plásticas pós-consumidas no meio ambiente tem representado uma preocupação crescente da sociedade em função de seu alto volume, conseqüente descarte e lenta degradação ${ }^{[1]}$. As poliolefinas, principalmente polietileno, são os materiais plásticos encontrados em maior quantidade nos resíduos urbanos. O grande interesse no reaproveitamento desses polímeros tem levado ao desenvolvimento de várias técnicas de reciclagem, dando origem a materiais reciclados com diversas aplicações práticas, como seu emprego para a obtenção de compósitos $^{[2]}$.

Fibras naturais têm sido cada vez mais utilizadas como reforçadores para termoplásticos, devido ao baixo custo da matéria-prima, sua biodegradabilidade e pelas boas propriedades mecânicas conferidas ao compósito ${ }^{[3]}$. Além disso, o desenvolvimento de compósitos de polímeros

Autor para correspondência: Claudia M. C. Bonelli, Departamento de Engenharia Mecânica e de Materiais, Instituto Militar de Engenharia, Praça General Tibúrcio 80, CEP:22290-270, Rio de Janeiro, RJ. E-mail: cmcb@openlink.com.br 
sintéticos com fibras naturais contribui para a redução da poluição ambiental, bem como para a criação de novos empregos e para a utilização de recursos naturais disponíveis ${ }^{[4]}$. Atualmente as fibras naturais mais utilizadas na produção de compósitos são: juta, rami, linho e sisal ${ }^{[5-7]}$. Madeira, sob a forma de serragem, também tem sido empregada na fabricação desses materiais ${ }^{[8]}$. Fibras de piaçava, da família Palmae, vêm sendo bastante empregadas na fabricação de produtos simples, tais como escovas, vassouras, cordas, entre outros, e possuem alto teor de lignina $(\sim 45 \%)^{[9,10]}$

A exploração de piaçaveiras no Brasil é uma atividade extrativista, tanto na Amazônia quanto na Bahia; algumas comunidades de baixa renda das regiões Norte e Nordeste dependem da extração dessas fibras naturais para sobreviverem.

O País produziu cerca de 9.300 toneladas de fibras de piaçava em 2001, boa parte das quais exportada para diversos países (Portugal, Alemanha, Estados Unidos e Holanda), que a utilizam na fabricação de escovas para máquinas de varrer rua e vassouras de uso doméstico ${ }^{[11]}$.

Foi reportado na literatura o uso de piaçava como reforço em matrizes de poliéster insaturado ${ }^{[10]}$ e polietileno de alta densidade reciclado ${ }^{[12]}$. Os resultados mostraram que a fibra de piaçava pode ser empregada como reforçadora em compósitos de matriz polimérica ${ }^{[10,12]}$. Foi reportado que compósitos de poliéster insaturado com fibra de piaçava podem substituir a madeira em aplicações tradicionais ${ }^{[10]}$. Foi também investigada a incorporação de fibra de curauá em polipropileno reciclado. A adição dessa fibra natural provocou aumento linear de $70 \%$ do módulo de elasticidade sob tração e queda brusca no alongamento na ruptura em função do aumento do teor de fibra ${ }^{[13]}$.

As propriedades mecânicas dos compósitos dependem de diversos fatores, tais como o tipo, volume, orientação, dispersão da fibra curta na matriz polimérica, bem como a afinidade química entre fibra e matriz. A melhora das propriedades do produto final pode ser conseguida através da incorporação de fibras tratadas superficialmente com agentes de acoplamento a fim de aumentar a adesão interfacial entre fibra e matriz ${ }^{[14,15]}$.

No presente trabalho foram avaliadas as propriedades térmicas, mecânicas (tração e flexão) e morfológicas de compósitos de HDPE reciclado (HDPE-r) e fibra de piaçava da Amazônia (Leopoldinia piassaba), tratada e não-tratada, em diferentes proporções (5, 10 e $15 \%$ de reforço). As fibras foram submetidas a tratamento superficial com silano de duas formas: incorporação direta e por solução metanólica do agente de acoplamento.

\section{Experimental}

O polímero reciclado, obtido a partir de embalagens de óleo lubrificante pós-consumidas, era proveniente da empresa LMG Indústria e Comércio de Plásticos. Imediatamente antes do processamento os pellets eram secos em estufa com circulação de ar a $100{ }^{\circ} \mathrm{C}$ por $1 \mathrm{~h}$. O HDPE-r apresentava índice de fluidez de $0,3 \mathrm{~g} / 10 \mathrm{~min}$, de acordo com a norma ASTM 1238. A densidade do material foi determinada de acordo com a norma ASTM D792, utilizando-se como solvente o etanol. A temperatura de fusão cristalina do polímero reciclado $\left(\mathrm{T}_{\mathrm{m}}\right)$ foi obtida em calorímetro diferencial de varredura Perkin Elmer, com taxa de aquecimento de $10{ }^{\circ} \mathrm{C} / \mathrm{min}$.

Fibras de piaçava in natura, provenientes da região de São Gabriel da Cachoeira (AM), com comprimento variando entre 0,40 a $1,80 \mathrm{~m}$, foram recebidas sob a forma de fardos. Os feixes foram fragmentados em picotador de facas rotatórias Brabender, para obtenção de $500 \mathrm{~g}$ de fibras de piaçava curta $(1-4 \mathrm{~mm})$. A sua distribuição granulométrica foi realizada em peneirador vibratório de laboratório tipo PSA, de acordo com norma DIN 4188. Fragmentos com diâmetro entre 0,25 e $0,71 \mathrm{~mm}$ foram lavados em água corrente e secos em estufa com circulação de ar a $100{ }^{\circ} \mathrm{C}$ por $24 \mathrm{~h}$. Em seguida, as fibras foram tratadas com 3-metacriloxipropil-trimetoxi-silano (D’Altomare, São Paulo, SP) por 2 diferentes métodos: no primeiro método, foram imersas em $100 \mathrm{ml}$ de solução 5\% $\mathrm{m} / \mathrm{m}$ de silano em metanol e impregnadas a vácuo com a solução, durante 30 minutos (fibra-tms). As fibras ficaram imersas em $150 \mathrm{ml}$ de solução metanólica à temperatura ambiente até evaporação do solvente. Foram em seguida secas em estufa com circulação de ar a $100^{\circ} \mathrm{C}$ por $5 \mathrm{~h}$. No segundo método, as fibras foram impregnadas diretamente por agitação manual com silano na proporção $1 / 3(\mathrm{p} / \mathrm{p})$ silano / fibra (fibra-ts) ${ }^{[16]}$. Compósitos de polímero reciclado com fibra tratada ou não tratada (fibra-nt), nas proporções 95/5; 90/10 e 85/15 foram obtidos em câmara de mistura Haake a $160^{\circ} \mathrm{C}$ por $5 \mathrm{~min}$ com rotação de $60 \mathrm{rpm}$. Placas de $15 \mathrm{~cm}$ de lado foram moldadas por compressão em prensa hidráulica Carver à temperatura de $190^{\circ} \mathrm{C}$ e pressão de $0,24 \mathrm{MPa}$ por 5 min e, em seguida, foram submetidas a rápido resfriamento em banho de água e gelo (temperatura de $10^{\circ} \mathrm{C}$ ) por $10 \mathrm{~min}$. A análise termogravimétrica (TGA) das matérias primas e dos compósitos foi realizada em equipamento Shimadzu TGA-50 em amostras de aproximadamente $10 \mathrm{mg}$ em atmosfera de nitrogênio, com taxa de aquecimento de $20^{\circ} \mathrm{C} / \mathrm{min}$. Corpos de prova para realização de ensaios de flexão e tração foram obtidos de acordo com as normas ASTM D790 e D638, respectivamente, a partir das placas moldadas. Os ensaios mecânicos foram realizados em máquina universal de ensaios Instron, com célula de $1 \mathrm{kN}$ e velocidades de teste de $1,7 \mathrm{~mm} / \mathrm{min}$ e $10 \mathrm{~mm} / \mathrm{min}$ para os ensaios de flexão e tração, respectivamente. A análise morfológica foi realizada em microscópio eletrônico de varredura modelo JSM 5800LV JEOL a partir de amostras de compósito fraturadas em nitrogênio líquido e cobertas com fina camada de ouro.

\section{Resultados e Discussão}

Os valores obtidos de temperatura de fusão cristalina $\left(132{ }^{\circ} \mathrm{C}\right)$ e densidade $\left(0,96 \mathrm{~g} / \mathrm{cm}^{3}\right)$ para o polímero reciclado indicaram que o material era constituído predominantemente por polietileno de alta densidade. 


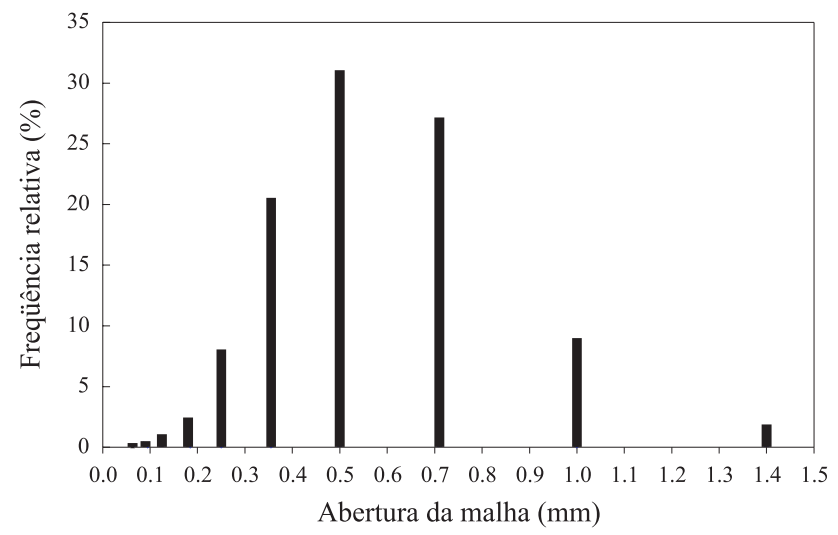

Figura 1. Distribuição granulométrica das fibras de piaçava

Os processos de fragmentação, lavagem e secagem das fibras produziram uma perda de $10 \%$ em massa, tendo em vista a heterogeneidade e contaminação da fibra por resíduos como terra, poeira, folhas, entre outros. A Figura 1 apresenta a distribuição granulométrica dos fragmentos da piaçava. Observa-se que a maior incidência se encontra na faixa de 0,25 a $0,71 \mathrm{~mm}$ de diâmetro de fibra, a qual foi utilizada para a confecção dos compósitos.

A Figura 2 apresenta as curvas de análise termogravimétrica (TGA) do polímero, da fibra e dos compósitos com fibra de piaçava não tratada. Podem-se observar as perdas de massa relativas ao processo de degradação da fibra de piaçava, especialmente pirólise dos seus constituintes principais: hemicelulose, celulose e lignina ${ }^{[17]}$. O onset mostra a derivada da curva de TGA. Abaixo de $200^{\circ} \mathrm{C}$ a perda de massa equivalente ao percentual de umidade da fibra que foi de aproximadamente $5 \%$; valor menor que o encontrado para sisal $(\sim 11 \%)$, mas comparável àquele encontrado para o linho ${ }^{[18]}$. As curvas do onset na Figura demonstram que ocorreu leve diminuição na estabilidade térmica do HDPE-r após adição da fibra em diferentes composições (HDPE-r, $496{ }^{\circ} \mathrm{C}$; HDPE$\mathrm{r} /$ fibra-nt $95 / 5,486{ }^{\circ} \mathrm{C}$; HDPE-r / fibra-nt $90 / 10,489{ }^{\circ} \mathrm{C}$; HDPE-r / fibra-nt $85 / 15,492{ }^{\circ} \mathrm{C}$ ), o que não chega a comprometer o processamento e moldagem de placas dos compósitos a temperaturas de 160 e $190{ }^{\circ} \mathrm{C}$, respectivamente.

A Figura 3 apresenta as curvas de TGA para os compósitos

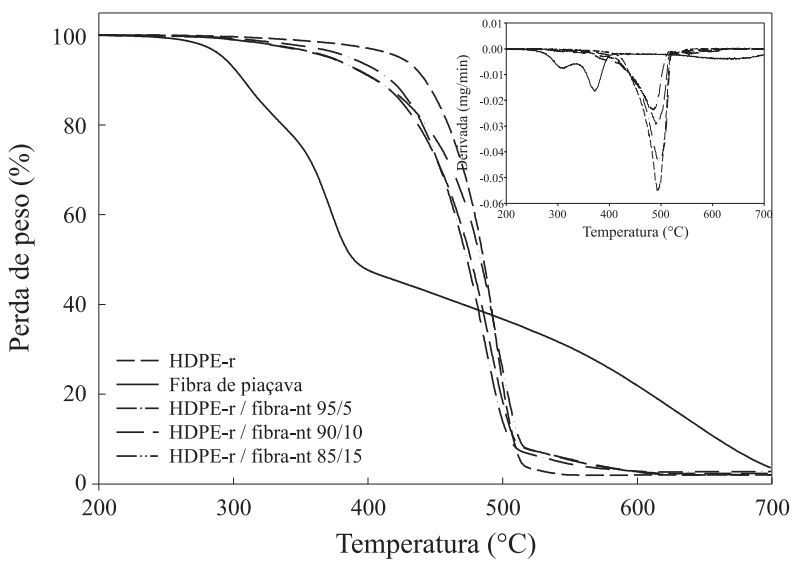

Figura 2. Curvas de TGA das matérias primas e dos compósitos HDPE-r / fibra em diferentes composições

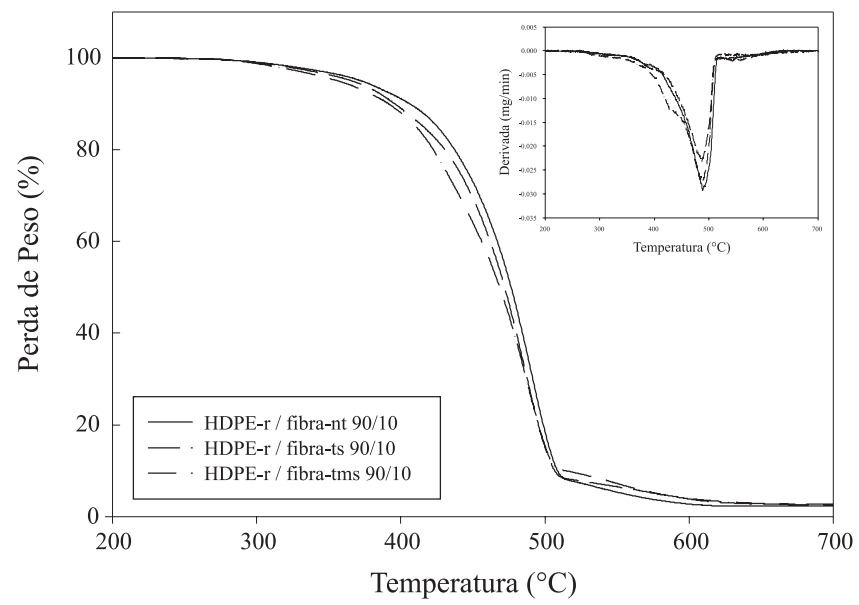

Figura 3. Curvas de TGA dos compósitos HDPE-r / fibra 90/10

de HDPE reciclado / fibra na composição 90/10. Estes termogramas mostram que o início do processo da degradação é influenciado pelo tratamento específico aplicado à fibra. É possível observar que há uma pequena redução da estabilidade térmica do compósito HDPE-r / fibra t-ms em relação ao compósito HDPE-r / fibra t-s.

A Figura 4 ilustra o efeito da quantidade de fibras tratadas e não-tratadas na resistência à flexão em $5 \%$ de deformação do compósito HDPE reciclado / fibra de piaçava. A adição de fibra não-tratada à matriz polimérica não ocasionou aumento na resistência à flexão. Comportamento semelhante foi observado na incorporação de fibra tratada com solução metanólica de silano, devido a uma provável extração da fração lignânica da fibra pelo metano[ ${ }^{[19]}$. Esta fração lignânica é responsável possivelmente pelo aumento do reforço estrutural da fibra, assim como pela melhor interação entre $o$ agente de acoplamento e a mesma ${ }^{[3]}$.

A adição de fibra tratada diretamente com silano promoveu um aumento na resistência à flexão do compósito, em especial no composto com $15 \%$ de fibra. Os resultados do módulo de flexão confirmam este comportamento. Os compósitos confeccionados com fibra tratada com silano apresentaram-se mais duros e resistentes à flexão com 5\% de deformação que os demais, conforme esperado, uma vez

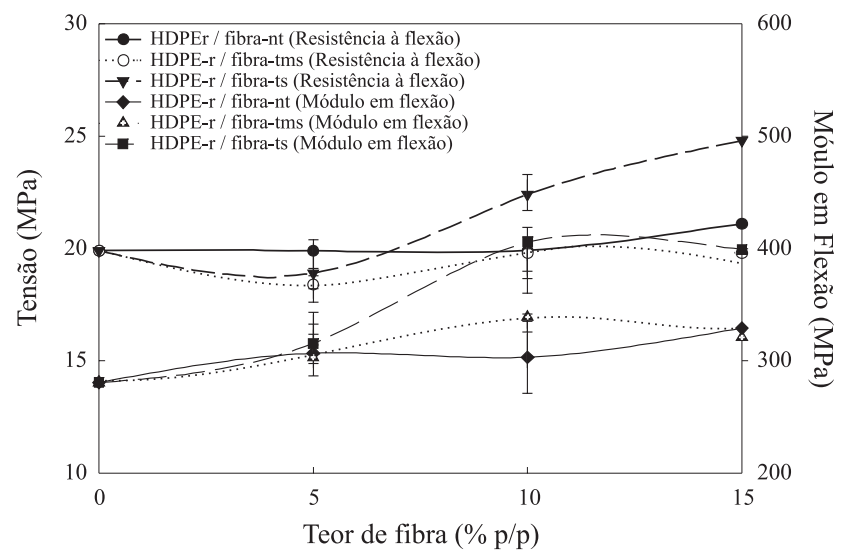

Figura 4. Resistência à flexão do compósito HDPE-r / fibra de piaçava em função do percentual de fibra 


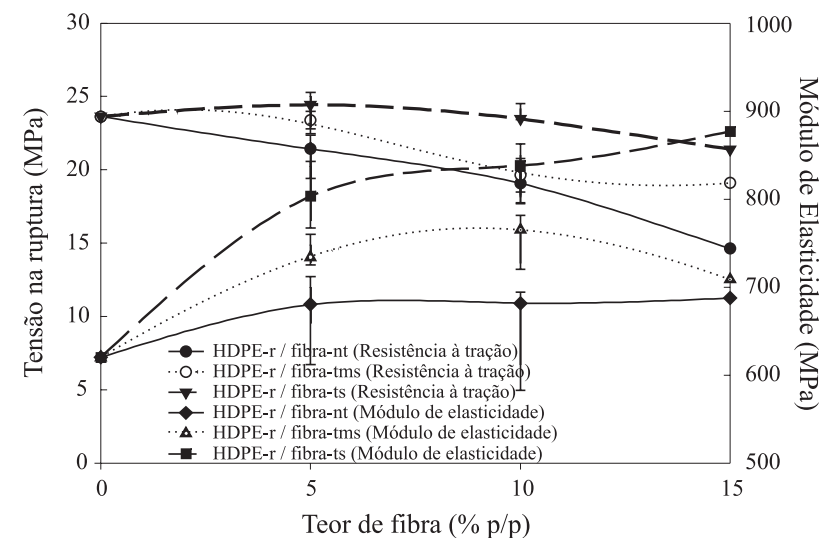

Figura 5. Resistência à tração do compósito HDPE-r/ fibra de piaçava em função do percentual de fibra

que ocorreu provavelmente um aumento de adesão interfacial entre fibra e matriz.

Vale ressaltar que as fibras de piaçava exibiam comprimento de $1-4 \mathrm{~mm}$ antes da mistura com o polímero; durante o processamento, as fibras são em geral quebradas em menores fragmentos, o que as torna muito curtas para o uso como reforço, conforme reportado na literatura. Uma vez que o comprimento crítico da fibra depende da transferência de esforço entre a matriz e a fibra, uma forma de superar esse problema é o aumento da adesão interfacial entre as fibras e o polímero ${ }^{[20]}$.

A Figura 5 mostra o efeito da percentagem de fibras tratadas e não-tratadas na resistência à tração de HDPE reciclado. A adição de fibra não-tratada não só reduziu significativamente a tensão na ruptura assim como não alterou o módulo de elasticidade do HDPE-r. A fragilidade do material é provavelmente explicada pela concentração de tensões na interface entre fibra e matriz.

A adição de fibra tratada com solução metanólica de silano aumentou em pequena proporção o módulo de elasticidade do polímero, com redução da tensão na ruptura. A fibra tratada apenas com silano apresentou os melhores resultados quanto à tensão na ruptura e o módulo de elasticidade, corroborando o comportamento observado nos ensaios de flexão.

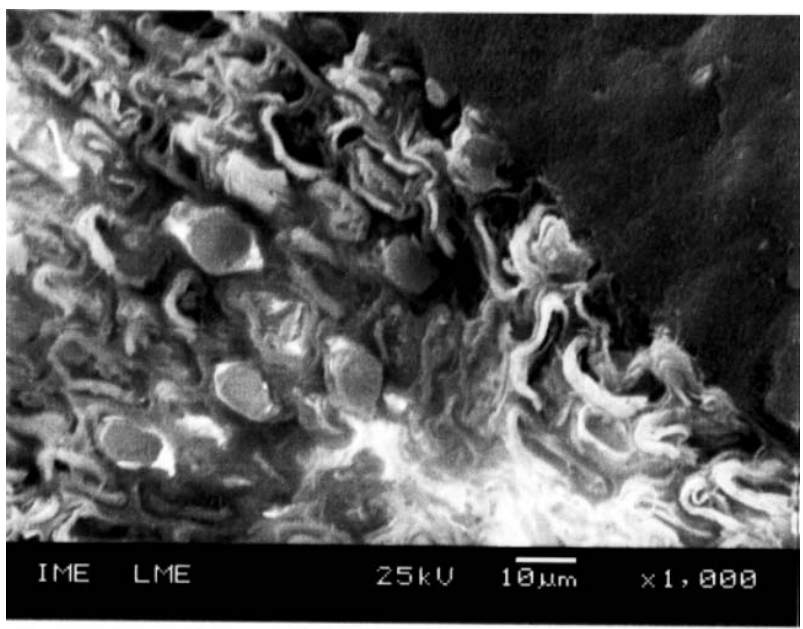

Figura 6. Microfotografia do compósito HDPE-r / fibra não tratada 85/15

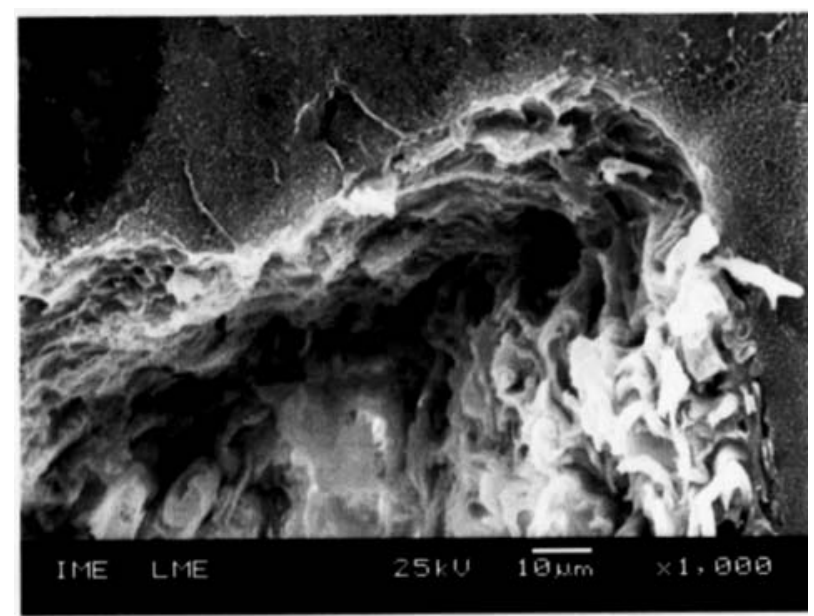

Figura 7. Microfotografia do compósito HDPE-r / fibra tratada com silano $85 / 15$

As Figuras 6 e 7 apresentam as micrografias dos compósitos HDPE-r / fibra-nt e fibra-ts, na proporção $85 / 15$, respectivamente. Verifica-se que na Figura 6 ocorre uma nítida separação entre a matriz e o reforço, resultante, provavelmente, da baixa compatibilidade entre os dois componentes. A Figura 7 mostra uma maior rugosidade na interface matriz-reforço, indicando que a adição de silano à fibra aumenta a compatibilidade entre HDPE-r e a piaçava e, em conseqüência, a adesão interfacial e as propriedades mecânicas.

\section{Conclusões}

Pode-se concluir que os compósitos de polietileno de alta densidade reciclado com fibra de piaçava tratada apenas com silano apresentaram melhor desempenho mecânico (resistência à flexão e à tração) que aquele obtido em compósitos com fibra tratada com solução metanólica e com fibra não tratada. O melhor desempenho mecânico do compósito com fibra tratada está associado ao aumento da adesão fibra-matriz de HDPE reciclado, conforme observado pelas micrografias apresentadas. Os melhores resultados foram obtidos com a incorporação de $15 \%$ de fibra tratada diretamente com silano.

Os resultados mostram que ocorreu leve diminuição na estabilidade térmica dos compósitos após adição de até $15 \%$ de fibra de piaçava, o que não chega a comprometer o processamento e moldagem de placas dos compósitos a temperaturas de 160 e $190^{\circ} \mathrm{C}$, respectivamente. Podemos concluir que os diferentes tratamentos não influenciaram drasticamente a estabilidade térmica dos compósitos. Prosseguem os estudos neste projeto.

\section{Agradecimentos}

Os autores agradecem às agências financiadoras FAPERJ, CNPq e CAPES, à LMG Indústria e Comércio de Plásticos, pelo fornecimento de plástico reciclado e ao Instituto Politécnico de Rio de Janeiro, UERJ (Nova Friburgo, RJ), pela determinação do índice de fluidez. 


\section{Referências Bibliográficas}

1. Bonelli, C.M.C. \& Mano, E.B. - Revista Gerenciamento Ambiental, São Paulo, SP, 3, p.48 (2001).

2. Dintcheva, N.T.; Jilov, N. \& Mantia, F.P. - Polym. Degrad. Stab., 57, p.191 (1997).

3. Mano, E. B. - "Polímeros como Materiais de Engenharia", Edgard Blucher, São Paulo (1991).

4. Leão, A.L.; Caraschi, J.C. \& Tan, I.H. - "Curaua fiber - a tropical natural fibers from Amazon - potential and applications in composites", in: Natural polymers and agrofibers based composites, E. Frollini, A.L. Leão e L.H.C. Mattoso (ed.), São Carlos (2000).

5. Benatti, R. - "Natural fibers in Brazil: utilization, perspectives and processing of sisal, ramie, jute, kenaf and allied fibers for composites production", in: Lignocellulosic-plastics composites, A.L. Leão, F.X. Carvalho e E. Frollini (ed.), USP/UNESP, São Paulo (1997).

6. Mano, E.B. - "Jute/Resin sand blocks for tropical housing", in: Lignocellulosic-plastics composites, A.L. Leão, F.X. Carvalho \& E. Frollini (ed.), USP/UNESP, São Paulo (1997).

7. Kuruvilla, J. \& Matoso, L.H.C. - "Sisal fibre reinforced polymer composites: status and future", in: Proceedings of $3^{\text {th }}$ International Symposium on Natural Polymers and Composites, p.333, São Carlos, SP (2000).

8. Coutinho, F.M.B.; Costa, T.H.S.; Suarez, J.C.M \& Melo, D.P. - Polym. Testing, 19, p. 625 (2000).

9. Mano, E.B.; Mendes, L.C.; Braga-Junior, E.; Chagas, B. \& Lopez, G.A.A.P. - Textília, 35, p.30 (2000).

10. Aquino, R.C.M.P; d'Almeida, J.R.M \& Monteiro, S.N. "Propriedades de compósitos de piaçava com matriz polimérica", in: $55^{\circ}$ Congresso Anual da ABM, p. 2898, Rio de Janeiro, RJ (2000)

11. Disponível em http://globorural.globo.com/barra.asp?d=/ edic/ 174/rep_piacava2.htm, capturado em 22 de outubro de 2002.
12. Bonelli, C.M.C.; A.Elzubair; H.R. Martins; J.C. Miguez Suarez \& E.B. Mano - "Utilização de fibras de piaçava como reforço em compósitos de matriz polimérica reciclada", in: $58^{\circ}$ Congresso Anual da ABM, p. 1778, Rio de Janeiro, RJ (2003).

13. Spinacé, M.A.S.; Allganer, K; Bastos, F.A.; Pedroso, A.G.; De Paoli, M.A. - "Efeito do teor e tratamento superficial de fibra curauá sobre as propriedades mecânicas de compósitos com polipropileno reciclado", in: $6^{0}$ Congresso Brasileiro de Polímeros, p.594, Gramado, RS (2001).

14. Marcovich, N; Reboredo, M.M. \& Aranguren, M.I. "Chemical modification of lignocellulosic materials. The utilization of natural fibers as polymer reinforcement", in: Lignocellulosic-plastics composites, A.L. Leão, F.X. Carvalho e E. Frollini (ed.), USP/UNESP, São Paulo (1997).

15. O'Conor, J. - Rubber Chemistry and Technology, 50, p. 945 (1977). $16 . \quad$ Hill, C.A.S. \& Abdul Khalil, H.P.S.- J. Appl. Polym. Sci., 78, p.1685 (2000).17. Grassie, N. \& Scott, G. - "Polymer Degradation and Stabilization", Cambridge University Press, Cambridge (1985).

16. Satyanarayana, K.G.; Sukumaran, K.; Mukherjee, P.S.; Pavithran, C. \& Pillai, S.G. -Cement \& Concrete Composites, 12, p.117 (1990).

17. Santos, M.S. - "Propriedades térmicas e mecânicas de materiais reciclados à base de PET pós-consumo e cargas de coco", Tese de Mestrado, Universidade Federal do Rio de Janeiro, Brasil (2002).

18. Gtenholm, P - "Interfacial adhesion and dispersion in biobased composites. Molecular interactions between cellulose and other polymers", in: Lignocellulosicplastics composites, A.L. Leão, F.X. Carvalho e E. Frollini (ed.), USP/UNESP, São Paulo (1997).

Enviado: 02/12/04 Reenviado: 04/07/05 Aprovado: 12/07/05 